\title{
Evaluating the performance characteristics of different antimicrobial susceptibility testing methodologies for testing susceptibility of gram-negative bacteria to tigecycline
}

\author{
Sima Babaei and Mehri Haeili ${ }^{*}$ iD
}

\begin{abstract}
Background: The current emergence of multi-drug resistance among nosocomial pathogens has led to increased use of last-resort agents including Tigecycline (TGC). Availability of reliable methods for testing TGC susceptibility is crucial to accurately predict clinical outcomes. We evaluated the influence of different methodologies and type of media on TGC susceptibility of different gram-negative bacteria of clinical origin.

Methods: The TGC susceptibility of 84 clinical isolates of Klebsiella pneumoniae $(n=29)$, Escherichia coli $(n=30)$, and Acinetobacter baumannii $(n=25)$ was tested by broth microdilution (BMD), Etest, agar dilution (AD) and disk diffusion (DD) methods using Mueller Hinton agar from Difco and Mueller Hinton broth (MHB) from two different manufacturers (Difco and Condalab). FDA TGC susceptibility breakpoints issued for Enterobacteriaceae were used for interpretation of the results.

Results: MICs determined by BMD using MHB from two suppliers showed a good correlation with overall essential agreement (EA) and categorical agreement (CA) being 100\% and 95\% respectively. However, a twofold rise in BMDCondalab MICs which was detected in $50 \%$ of the isolates, resulted in changes in susceptibility categories of few isolates with MICs close to susceptibility breakpoints leading to an overall minor error (MI) rate of $4.7 \%$. Among the tested methods, Etest showed the best correlation with BMD, being characterized with the lowest error rates (only $1 \% \mathrm{MI})$ and highest overall EA (100\%) and CA (98.8\%) for all subsets of isolates. AD yielded the lowest overall agreement (EA 77\%, CA 81\%) with BMD in a species dependent manner, with the highest apparent discordance being found among the A. baumannii isolates. While the performance of DD for determination of TGC susceptibility among Enterobacteriaceae was excellent, (CA:100\% with no errors), the CA was lower (84\%) when it was used for A. baumannii where an unacceptably high minor-error rate was noted (16\%). No major error or very major error was detected for any of the tested methods.
\end{abstract}

Conclusions: Etest can be reliably used for TGC susceptibility testing in the three groups of studied bacteria. For the isolates with close-to-breakpoint MICs, testing susceptibility using the reference method is recommended.

Keywords: Tigecycline, Broth microdilution, Etest, Agar dilution, Disk diffusion, Gram-negative bacteria

\footnotetext{
*Correspondence: m.haeili@tabrizu.ac.ir; m.haeili@yahoo.com

Department of Animal Biology, Faculty of Natural Sciences, University of

Tabriz, Tabriz, Iran
}

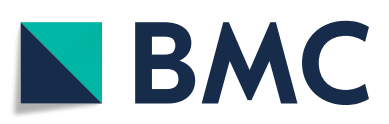

C C The Author(s). 2021 Open Access This article is licensed under a Creative Commons Attribution 4.0 International License, which permits use, sharing, adaptation, distribution and reproduction in any medium or format, as long as you give appropriate credit to the original author(s) and the source, provide a link to the Creative Commons licence, and indicate if changes were made. The images or other third party material in this article are included in the article's Creative Commons licence, unless indicated otherwise in a credit line to the material. If material is not included in the article's Creative Commons licence and your intended use is not permitted by statutory regulation or exceeds the permitted use, you will need to obtain permission directly from the copyright holder. To view a copy of this licence, visit http://creativecommons.org/licenses/by/4.0/ The Creative Commons Public Domain Dedication waiver (http://creativecommons.org/publicdomain/zero/1.0/) applies to the data made available in this article, unless otherwise stated in a credit line to the data. 


\section{Introduction}

Tigecycline (TGC) is a new semisynthetic glycylcyclin with expanded-spectrum antibacterial activity against gram-negative and gram-positive bacteria [1]. It retains activity against most clinically significant multi-drug re- in four hospitals located in different regions of Iran. Identification of the isolates to species level was performed by conventional biochemical tests [15], detection

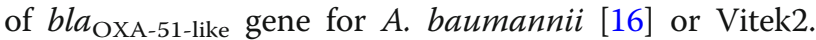
Some of the tigecycline non-susceptible isolates were sistant (MDR) gram-negative bacteria (GNB) including extensively drug resistant $A$. baumannii [2] and carbapenem resistant Enterobacteriaceae (CRE) [3, 4]. Tigecycline is approved by the FDA for complicated skin and skin structure infections, complicated intraabdominal infections, and community-acquired bacterial pneumonia [5]. TGC inhibits bacterial protein synthesis and is not affected by the known tetracycline resistance determinants (ribosomal protection and TetA-E Efflux pumps) [6, 7]. Increased administration of TGC in clinical settings has resulted in the development of resistance to this last resort antibiotic among the difficult to treat pathogens. The mechanism of resistance to TGC primarily involves overexpression of RND family efflux pumps $[8,9]$, or it can be mediated by other determinants including rps [5] and tetA mutations [10] or by enzymatic inactivation by TetX variants [11]. Despite being used clinically since 2005, TGC susceptibility testing is still considered challenging. There are currently no defined CLSI susceptibility breakpoints for tigecycline and EUCAST TGC breakpoints are only available for a handful of GNBs (E. coli and C. koseri). It has been demonstrated that the TGC susceptibility testing outcomes can be influenced by different factors including the chosen testing methodology and the manganese content or the age of the medium used for antimicrobial susceptibility testing (AST) [12-14]. The uptick in clinical use of tigecycline necessitates availability of reliable susceptibility testing methods as alternatives to the standard broth microdilution method. A simpler assay that could reliably test TGC susceptibility such as disk diffusion may be the only option in resource-limited clinical laboratories where performance of an ideal standardized microdilution assay is not practical. Similarly, Etest strips are a convenient alternative to conventional dilution-based susceptibility testing methods. The aim of this study was to assess the influence of different methodologies and type of medium on TGC susceptibility of different gramnegative bacteria of clinical origin.

\section{Materials and methods}

\section{Bacterial isolates}

The study included 84 clinical isolates of Escherichia coli $(n=30)$, Klebsiella pneumoniae $(n=29)$ and A. baumannii $(n=25)$ recovered during 2015 to 2020 from patients intentionally included from our previous works ( [17, 18 ] to have a complete series of susceptible (S), Intermediate (I) or resistant (R) isolates to assess the performance of different testing methods for correct identification of all three susceptibility categories. E. coli ATCC 25922 was used as quality control strain for antimicrobial susceptibility testing.

\section{Susceptibility testing}

MICs were determined in triplicate by broth microdilution testing using freshly prepared (less than $12 \mathrm{~h}$ old) Mueller Hinton broth (MHB) from Difco (BD Diagnostic Systems, Sparks, MD) (lot 8,170,915) and Condalab (Madrid, Spain) (CAT:1214.00, Batch No. 802083). TGC concentrations (Glentham Life Sciences (UK) (batch No.176ZIJ and 389SOI) spanned a doubling dilution range of $0.015 \mathrm{mg} / \mathrm{l}$ to $64 \mathrm{mg} / \mathrm{l}$. MICs were recorded after incubation at $35-37^{\circ} \mathrm{C}$ in a non- $\mathrm{CO}_{2}$ incubator for 16 to $20 \mathrm{~h}$ as the lowest concentration of the agent that inhibited visible growth of the tested isolate as judged by the naked eye. Etest, disk diffusion and agar dilution were all performed using the Mueller-Hinton agar (MHA) from Difco (BD Diagnostic Systems, Sparks, MD) (lot 7,271,779) which have been previously determined to have a lower manganese content compared to other brands [13]. Agar diffusion methods were performed using Liofilchem Etest Strips (Liofilchem, Roseto degli Abruzzi, Italy) code 92144) containing concentration gradient range of TGC (0.016 to $256 \mathrm{mg} / \mathrm{l})$ or disks containing $15 \mu \mathrm{g}$ of TGC (Mast Co, Merseyside, UK) respectively according to the manufacturers' instructions with incubation at $35^{\circ} \mathrm{C}$ in the incubator for 16 to $24 \mathrm{~h}$. The Etest MIC endpoint was read where the growth inhibition ellipse intersected the MIC scale on the Etest strip. Agar dilution was performed by addition of appropriate amounts of $25,2.5$ or $0.25 \mathrm{mg} / \mathrm{ml}$ stock solutions of TGC to molten sterile MHA to provide concentrations ranging from 0.03 to $64 \mathrm{mg} / 1$ according to a method described previously [19]. MICs were recorded as the lowest concentration of the agent that inhibited visible growth of the tested isolate, disregarding the growth of a single colony or faint film caused by the inoculum. 


\section{Interpretation of results and data analysis}

Susceptibility results were compared and categorized in relation to BMD testing performed using MHB from Difco. Etest MIC values were rounded up to the next concentration of the standard doubling dilution scale when necessary. Categorical agreement (CA), essential agreement (EA), major errors (ME), very major errors (VME), and minor errors (MI) were evaluated as follow: EA was calculated by determining the number of test results that were within \pm 1 doubling dilution of the MIC obtained by BMD. CA was defined as the number of tests with correct susceptibility categorization between the method under evaluation and BMD. A very major error (VME) indicated a false-susceptible result; a major error (ME) indicated a false-resistant result; Minor errors (MI) were defined as one result yielding an intermediate category and the other either a susceptible or resistant result [20]. The statistical analyses were performed using MedCalc software, version 13 (MedCalc, Ostend, Belgium).

Due to the lack of established CLSI breakpoints for TGC at this time, Food and Drug Administration (FDA) breakpoints issued for Enterobacteriaceae, (susceptible $\leq 2 \mathrm{mg} / \mathrm{l}$, intermediate $=4 \mathrm{mg} / \mathrm{l}$, resistant $\geq 8 \mathrm{mg} / \mathrm{l}$ for MIC methods, and $\geq 19 \mathrm{~mm}$, susceptible; $15-18 \mathrm{~mm}$, intermediate; $\leq 14 \mathrm{~mm}$, resistant for disk diffusion) (https:// www.accessdata.fda.gov/drugsatfda_docs/label/2013/021 821s026s031lbl.pdf) were applied for interpretation of results. Since no TGC MIC breakpoints was available for Acinetobacter spp. we used the same breakpoints defined by the FDA for Enterobacteriaceae for interpretation of susceptibility testing results obtained for A. baumannii.

\section{Results}

The TGC MIC measurements for the tested ATCC reference strain were within the acceptable quality control ranges as specified by the CLSI (BMD-Difco: $0.06 \mathrm{mg} / \mathrm{l}$, BMD-Condalab: $0.12 \mathrm{mg} / \mathrm{l}$, E-test: $0.06 \mathrm{mg} / \mathrm{l}$, AD: 0.12 $\mathrm{mg} / \mathrm{l}$, DD: $25 \mathrm{~mm}$ ). According to TGC susceptibility testing results obtained by BMD (Difco) as reference method, 68 isolates (30 E. coli, 19 K. pneumoniae and 19 A. baumannii) were TGC-susceptible and the remaining 16 isolates were found to be TGC non-susceptible ( $R$ $(n=9)$ or I $(n=7))$ which included $10 \mathrm{~K}$. pneumoniae ( $n=8$ resistant, $n=2$ intermediate) and 6 A. baumannii ( $n=1$ resistant, $n=5$ intermediate). Since some of the TGC-I or TGC-R isolates were included in the study on purpose, the obtained data does not reflect the true efficacy of TGC on the tested bacterial isolates. The TGC MIC distributions of the E. coli, K. pneumoniae and A. baumannii ranged from 0.1 to $1 \mathrm{mg} / \mathrm{l}, 0.2-32 \mathrm{mg} / \mathrm{l}$ and < $0.1-8 \mathrm{mg} / \mathrm{l}$ respectively. Table 1 and Fig. 1 show the TGC MIC distribution of the tested isolates determined using the different AST methods.

\section{Comparison of BMD results performed by MHB from two different manufacturers}

Overall, for half of the strains (50\%), MICs determined by MHB from Condalab were 2-fold dilutions higher than those determined by BMD from Difco. Despite this,

Table 1 Tigecycine MICs of isolates obtained by different testing methodologies or medium types

\begin{tabular}{|c|c|c|c|c|c|c|c|c|c|c|c|c|}
\hline \multirow[b]{2}{*}{ Organism (n) } & \multirow[b]{2}{*}{ Method } & \multicolumn{11}{|c|}{ Number of isolates with MICs (mg I ${ }^{-1}$ ) } \\
\hline & & $<0.1$ & 0.1 & 0.2 & 0.5 & 1 & 2 & 4 & 8 & 16 & 32 & 64 \\
\hline \multirow[t]{4}{*}{ All isolates (84) } & BMD1 & 2 & 10 & 20 & 13 & 19 & 4 & 7 & 7 & 1 & 1 & 0 \\
\hline & BMD2 & 0 & 6 & 19 & 12 & 14 & 15 & 7 & 7 & 3 & 1 & 0 \\
\hline & Etest & 2 & 15 & 24 & 8 & 12 & 7 & 8 & 7 & 1 & 0 & 0 \\
\hline & $A D$ & 0 & 1 & 11 & 17 & 16 & 13 & 11 & 2 & 9 & 3 & 1 \\
\hline \multirow[t]{4}{*}{ K. pneumoniae (29) } & BMD1 & 0 & 0 & 3 & 4 & 10 & 2 & 2 & 6 & 1 & 1 & 0 \\
\hline & BMD2 & 0 & 0 & 2 & 2 & 6 & 7 & 2 & 6 & 3 & 1 & 0 \\
\hline & Etest & 0 & 0 & 6 & 5 & 7 & 1 & 3 & 6 & 1 & 0 & 0 \\
\hline & $A D$ & 0 & 0 & 0 & 2 & 6 & 9 & 2 & 2 & 7 & 0 & 1 \\
\hline \multirow[t]{4}{*}{ E. coli (30) } & BMD1 & 0 & 7 & 14 & 8 & 1 & 0 & 0 & 0 & 0 & 0 & 0 \\
\hline & BMD2 & 0 & 3 & 13 & 9 & 4 & 1 & 0 & 0 & 0 & 0 & 0 \\
\hline & Etest & 1 & 10 & 16 & 2 & 1 & 0 & 0 & 0 & 0 & 0 & 0 \\
\hline & $A D$ & 0 & 1 & 8 & 11 & 9 & 1 & 0 & 0 & 0 & 0 & 0 \\
\hline \multirow[t]{4}{*}{ A. baumannii (25) } & BMD1 & 2 & 3 & 3 & 1 & 8 & 2 & 5 & 1 & 0 & 0 & 0 \\
\hline & BMD2 & 0 & 3 & 4 & 1 & 4 & 7 & 5 & 1 & 0 & 0 & 0 \\
\hline & Etest & 1 & 5 & 2 & 1 & 4 & 6 & 5 & 1 & 0 & 0 & 0 \\
\hline & $A D$ & 0 & 0 & 3 & 4 & 1 & 3 & 9 & 0 & 2 & 3 & 0 \\
\hline
\end{tabular}



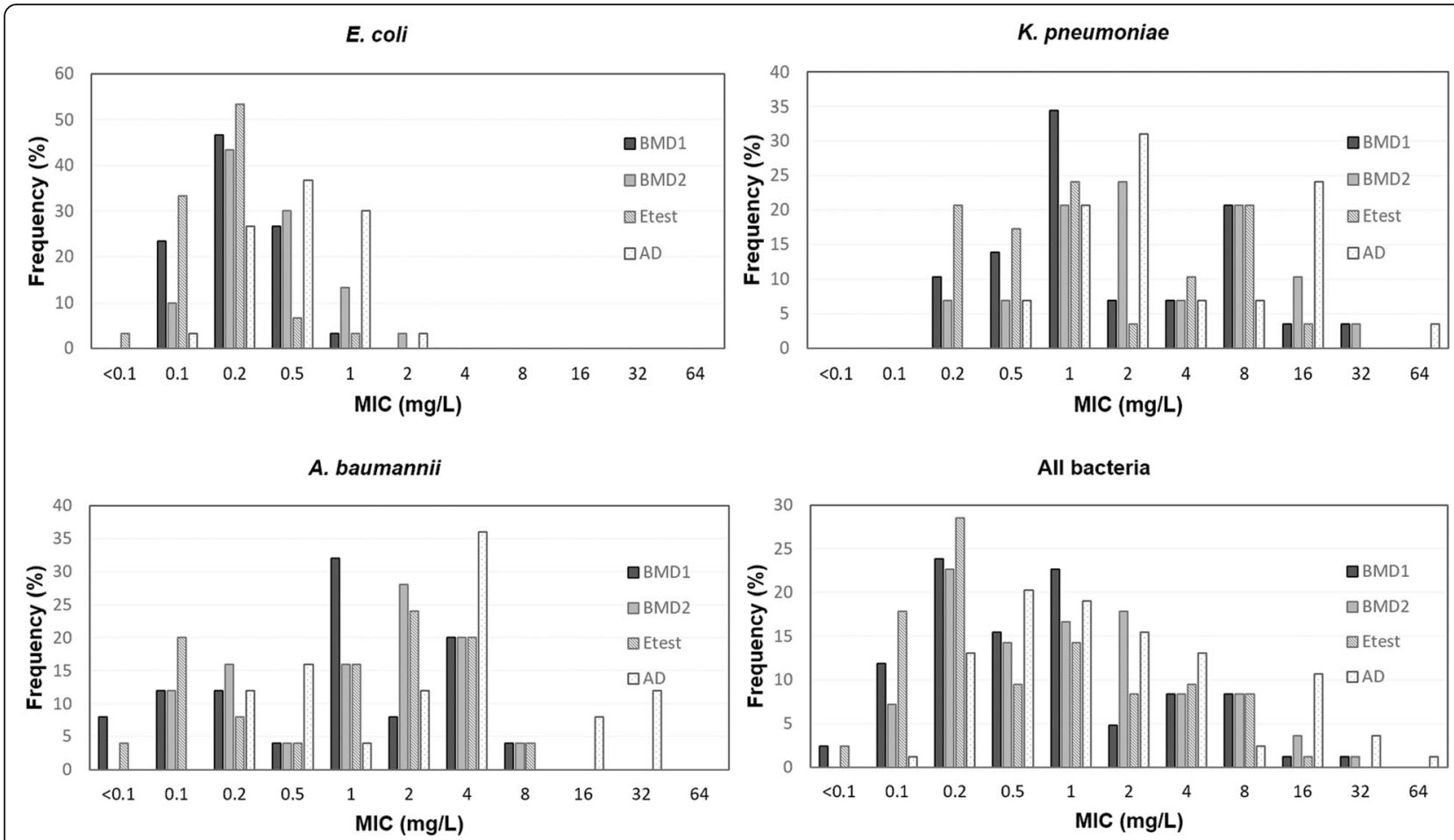

Fig. 1 Distribution of MICs determined by four different dilution-based methods for all subsets of isolates (BMD1, Broth microdilution -Difco; BMD2, Broth microdilution -Condalab, AD, Agar dilution)

a good correlation was found for MIC values obtained by MHB from two suppliers with EA and CA being 100 and $95 \%$ respectively (Weighted kappa $=0.911, \mathrm{SE}=$ $0.043,95 \% \mathrm{CI}=0.826-0.995)$. There was no VME or ME and a $4.7 \%$ of MI was found which was derived from 4 K. pneumoniae with close-to-breakpoint MICs that fell in the $S(n=2, M I C=2 \mathrm{mg} / \mathrm{l})$ or $\mathrm{I}(\mathrm{n}=2$,$) category by$ BMD-Difco but were categorized as I or R by MHB from Condalab respectively.

\section{Evaluation of performance of Etest in relation to BMD- Difco}

Comparison of TGC MIC results obtained by Etest showed a high level of agreement with those obtained using BMD (CA 98.8\%, EA 100\%) (Weighted kappa = 0.976, $\mathrm{SE}=0.024,95 \% \mathrm{CI}=0.929-1.00)$. Overall, Etest MIC values were either identical (61.9\% isolates, $n=52$ isolates, $17 \mathrm{E}$. coli, $18 \mathrm{~K}$. pneumoniae, $17 \mathrm{~A}$. baumannii) or within $-1(29.7 \%, n=25$ isolates, $12 \mathrm{E}$. coli, $11 \mathrm{~K}$. pneumoniae, $2 \mathrm{~A}$. baumannii) or +1 doubling dilution (8.3\%, $n=7$ isolates, $6 \mathrm{~A}$. baumannii, $1 \mathrm{E}$. coli) of the BMD MICs. There were no categorical discrepancies between the two testing methodologies except for one $K$. pneumoniae isolate with close-to-breakpoint MIC (=8 $\mathrm{mg} / \mathrm{l}$ ) which was categorized as R by BMD but I by the Etest resulting in overall MI rate of $1.19 \%$ (Table 2).

\section{Evaluation of performance of agar dilution in relation to BMD-Difco}

Overall, for $91.6 \%$ of strains $(n=77)$ MICs determined by agar dilution were at least 2-fold higher than those determined by reference BMD resulting in poor correlation between two methods (overall EA:77\%, CA:81\%) (Weighted kappa $=0.697, \mathrm{SE}=0.067,95 \% \mathrm{CI}=0.566-$ 0.829 ) and unacceptably higher MI rate (19\%). Despite the poor intermethod agreement, no ME or VME was detected (Table 2). The agreement between two methods differed on a species dependent manner, exceeding the acceptable performance rate for AST methods (>90\%) for K. pneumoniae and E. coli but being found unacceptable for $A$. baumannii isolates. Indeed, the performance of $\mathrm{AD}$ was the best for determination of MIC in $E$. coli where MICs were identical $(n=4),+1(n=24$ isolates $)$ or +2 ( $n=2$ isolates) doubling dilution of BMD MICs.

\section{Evaluation of performance of disk diffusion in relation to BMD-Difco}

Overall the inhibition zone diameter observed among the isolates ranged from 19 to $26 \mathrm{~mm}$ among TGC-S, $12-18 \mathrm{~mm}$ among TGC-I and $11-14 \mathrm{~mm}$ among TGC-R isolates. DD correctly detected all TGC-S $(n=68)$ and TGC-nonsusceptible $(n=16)$ isolates identified by reference method which resulted in high level overall CA between two methods (95\%) (Weighted kappa $=0.911$, 
Table 2 Performance characteristics of different TGC susceptibility testing methods compared to broth microdilution performed using Mueller Hinton Broth from Difco

\begin{tabular}{|c|c|c|c|c|c|c|}
\hline \multirow{2}{*}{$\begin{array}{l}\text { Method and } \\
\text { isolate group }\end{array}$} & \multicolumn{3}{|c|}{ No. (\%) of isolates } & \multirow[b]{2}{*}{ EA (\%) } & \multirow[b]{2}{*}{ CA (\%) } & \multirow[b]{2}{*}{ MI $n(\%)$} \\
\hline & Susceptible & Intermediate & Resistant & & & \\
\hline \multicolumn{7}{|l|}{ BMD1 } \\
\hline All isolates & $68(80.9)$ & $7(8.3)$ & $9(10.7)$ & & & \\
\hline K. pneumoniae & $19(65.5)$ & $2(6.8)$ & $8(27.5)$ & & & \\
\hline A. baumannii & $19(76)$ & $5(20)$ & $1(4)$ & & & \\
\hline E. coli & $30(100)$ & 0 & 0 & & & \\
\hline \multicolumn{7}{|l|}{ BMD2 } \\
\hline All isolates & $66(78.5)$ & $7(8.3)$ & $11(13)$ & 100 & 95.23 & $4(4.76)$ \\
\hline K. pneumoniae & 17 (58.6) & $2(6.8)$ & $10(34.4)$ & 100 & 86.20 & $4(13.7)$ \\
\hline A. baumannii & $19(76)$ & $5(20)$ & $1(4)$ & 100 & 100 & 0 \\
\hline E. coli & $30(100)$ & 0 & 0 & 100 & 100 & 0 \\
\hline \multicolumn{7}{|l|}{ Etest } \\
\hline All isolates & $68(80.9)$ & $8(9.5)$ & $8(9.5)$ & 100 & 98.80 & $1(1.19)$ \\
\hline K. pneumoniae & $19(65.5)$ & $3(10.3)$ & $7(24.1)$ & 100 & 96.55 & $1(3.4)$ \\
\hline A. baumannii & $19(76)$ & $5(20)$ & $1(4)$ & 100 & 100 & 0 \\
\hline E. coli & $30(100)$ & 0 & 0 & 100 & 100 & 0 \\
\hline \multicolumn{7}{|l|}{$A D$} \\
\hline All isolates & $58(69)$ & $11(13)$ & $15(17.8)$ & 77.38 & 80.95 & $16(19)$ \\
\hline K. pneumoniae & 17 (58.6) & $2(6.8)$ & $10(34.4)$ & 96.55 & 86.20 & $4(13.7)$ \\
\hline A. baumannii & $11(44)$ & $9(36)$ & $5(20)$ & 36 & 52 & $12(48)$ \\
\hline E. coli & $30(100)$ & 0 & 0 & 93.33 & 100 & 0 \\
\hline \multicolumn{7}{|l|}{ DD } \\
\hline All isolates & $68(80.9)$ & $3(3.5)$ & $13(15.4)$ & NA & 95.23 & $4(4.76)$ \\
\hline K. pneumoniae & $19(65.5)$ & $2(6.8)$ & $8(27.5)$ & & 100 & 0 \\
\hline A. baumannii & $19(76)$ & $1(4)$ & $5(20)$ & & 84 & $4(16)$ \\
\hline E. coli & 30 (100) & 0 & 0 & & 100 & 0 \\
\hline
\end{tabular}

NA Not applicable, EA Essential agreement, CA Categorical agreement, MI Minor error, BMD1 Broth microdilution -Difco, BMD2 Broth microdilution -Condalab, $A D$ Agar dilution, $D D$ Disk diffusion

$\mathrm{SE}=0.040,95 \% \mathrm{CI}=0.833-0.990)$. While the performance of DD for determination of TGC susceptibility among Enterobacteriaceae was excellent, (CA:100\% with no errors), the CA was slightly lower when this method was used for A. baumannii (84\%) where it was associated with an unacceptably high minor-error rate $(16 \%)$ (Table 2). All the minor errors were intermediate findings interpreted as resistant $(n=4$ intermediate isolates having inhibition zone diameter of less than $15 \mathrm{~mm}(12$ $\mathrm{mm}(n=1), 13 \mathrm{~mm}(n=1), 14 \mathrm{~mm}(n=2))$ (Fig. 2). This could be attributed to the fact that the susceptibility of A. baumannii was categorized based on the FDA susceptibility breakpoints for Enterobacteriaceae.

\section{Discussion}

The emergence of multi-drug resistance among nosocomial GNB greatly limits therapeutic options and necessitates the use of last resort antibiotics including TGC for the treatment of life-threatening infections. Therefore, there is an increased need for reliable methods to predict the clinical outcomes adequately. The TGC susceptibility testing is plagued by problems including the lack of predictive breakpoints for interpretation of the results in CLSI (although the EUCAST breakpoints are only available for few GNB (E. coli \& C. koseri)) and the variety of factors that can affect the susceptibility testing results. The U.S. FDA susceptibility breakpoints are used by many laboratories for interpretation of the results. However, there are no existing susceptibility breakpoints for some of the most important clinical pathogens including A. baumannii from any breakpoint organization, even in FDA. It has been shown that aged broth may lead to misleadingly higher TGC MIC values for some isolates and it is postulated that this may be due to inactivation by dissolved oxygen [12]. Therefore, it is recommended that broth MIC determinations for tigecycline should 


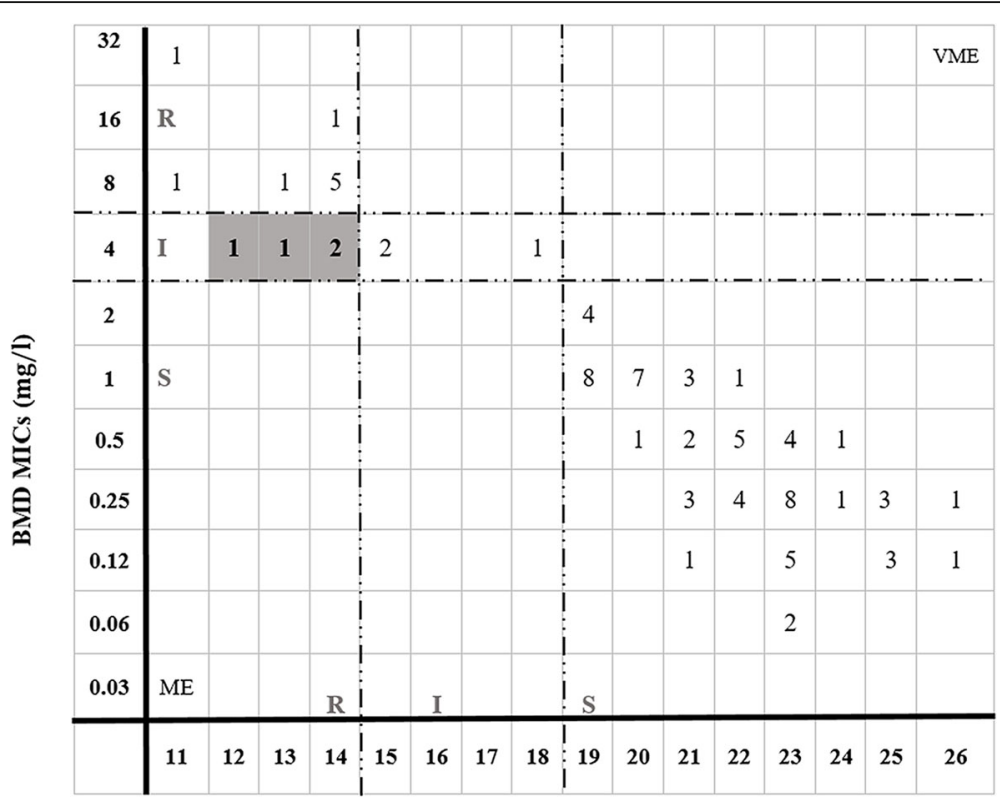

Disk diffusion zone diameter (mm)

Fig. 2 Scattergram of tigecycline zone diameters versus BMD MICs for 84 clinical isolates of gram-negative bacteria

always be performed using fresh media $(<12 \mathrm{~h}$ after autoclaving). Also a previous study found different MIC values obtained by Etest performed using MuellerHinton media from different manufacturers (Oxoid, BioRad, BD) for all tested organisms [14]. Others reported obtaining higher TGC MICs performed by Etest using Mueller-Hinton agar from Merck compared to MHA from either Oxoid or Difco. The differences were attributed to a much higher concentration of manganese in the medium from Merck compared to Difco or Oxoid [13]. We conducted the present study to assess the performance of different susceptibility testing methods against the reference BMD using the FDA breakpoints. All methods were performed using MHA/B from one manufacturer (Difco), except for BMD which was performed using MHB from two different suppliers (Difco and Condalab) to check the effect of medium type on TGC MICs. While comparing MICs obtained by BMD performed using MHB from two suppliers, revealed a good correlation (acceptable EA and CA), we found that MHB from Condalab produced a 2-fold dilution higher MICs than BMD-Difco for $50 \%$ of the isolates. This rise in MIC did not result in change in categoric susceptibility of most of the isolates except for those with MICs close to susceptibility breakpoints ( $\mathrm{MIC}=2$ or $4 \mathrm{mg} / \mathrm{l}$ ) which led to a slightly higher overall MI rate (4.7\%). The identified minor discordance between MICs obtained by using the two MHB media may be attributed to variation in manganese content of the medium from each manufacturer. Determination of manganese content of the medium using methods such as "inductively coupled plasma optical emission spectrometry" or "atomic absorption spectroscopy", was not possible in the current study and this information was not available from the Condalab company for the batch we used. However, it is speculated that, the higher MIC values obtained by MHB from Condalab may possibly be linked to a higher manganese concentration in this medium compared to reference medium. Among the studied methods, Etest showed the best correlation with BMD, being characterized with the lowest error rates and highest EA and CA. For the majority of isolates (91.6\%) Etest yielded either identical or one doubling dilution lower MICs than BMD MICs except for A. baumannii where Etest produced higher MICs among $24 \%$ of isolates. Other studies have reported obtaining higher or lower MICs given by Etest compared to MICs determined by BMD depending on bacterial isolate tested where higher Etest MICs were noted among A. baumannii isolates compared to BMD MICs [21]. Etest has also been reported in a previous study to yield $\geq 1$-fold dilution lower MIC values compared to BMD MICs when evaluated for testing TGC susceptibility of Enterobacteriaceae [22].

$\mathrm{AD}$ yielded the least correlation with BMD among the studied methods. This discordance was reflected in a low essential agreement between the two methods in a bacterial isolate dependent manner. The most apparent difference between the two testing methodologies was noted to occur among the A. baumannii, where a two ( $n=8$ isolates) to four fold ( $n=13$ isolates) or higher 
increase (eight fold, $n=3$ isolates) in AD MICs was found resulting in low $\mathrm{CA}$ and EA and higher MI relative to BMD. However, an acceptable EA $(96,93 \%)$ or slightly acceptable CA $(86,100 \%)$ was obtained when AD MICs were compared to BMD MICs among Enterobacteriaceae. Torrico et al., who tested the TGC susceptibility of Enterobacteriaceae using 3 different testing methodologies (BMD, Etest and $\mathrm{AD}$ ), also reported obtaining the highest $\mathrm{MIC}$ values by $\mathrm{AD}$ compared to other methods [22]. While DD showed excellent agreement with BMD in terms of correct categorization of all TGC-S, TGC-I and TGC- R isolates of $E$. coli and $K$. pneumoniae, it produced unacceptably higher minor error rate $(16 \%)$ for $A$. baumannii which probably stemmed from applying the FDA tigecycline breakpoints issued for Enterobacteriaceae for data interpretation in this bacterium. This is in agreement with a previous study which found higher MI for DD compared to BMD among ESBL-producing K. pneumoniae and A. baumannii isolates [23]. Moreover, testing the TGC susceptibility of Acinetobacter spp. by DD in another study also revealed a high minor-error rate (23.3\%) (by referring to FDA Enterobacteriaceae breakpoints for data interpretation). The adjustment of breakpoints (susceptible/resistant) to $\geq 16 / \leq 12 \mathrm{~mm}$ by the authors improved the intermethod agreement and minimized error rates down to $9.7 \%$ [24]. In fact, the validation of DD breakpoints for A. baumannii is needed to improve the performance and predictive value of this method. With a present global increase of TGC-resistance among $A$. baumannii isolates, performing a DD assay may be the most practical and available method in many laboratories, especially in those of developing countries.

In conclusion, with regard to BMD performed using MHB from Condalab and Etest we found MIC values which differed by one doubling dilution in 50 and 38\% of isolates from reference method MICs (BMD-Difco) respectively. Since these discrepancies in MICs can affect the clinical classification of isolates with close-tobreakpoint MICs, testing the susceptibility using a reference AST method or medium with optimized concentration of manganese is recommended if obtained MICs are found to be close to susceptibility breakpoints. Despite the lack of detected VME or ME, DD and $\mathrm{AD}$ should not be used for routine TGC susceptibility testing of A. baumannii, due to poor correlation with BMD. The higher intermethod MI error rate obtained for DD in $A$. baumannii isolates increasing a need for adjustment of susceptibility breakpoints by the breakpoint organizations. In general, all testing methods showed acceptable (>90\%) EA and/or CA with BMD in E. coli isolates which could stem from the fact that TGCs MIC ranges in these bacteria were very low and none of isolates were characterized with close-to-breakpoint MICs.

\section{Abbreviations}

TGC: Tigecycline; GNB: Gram-Negative bacteria; R: Resistant; I: Intermediate; S: Susceptible; TGC-S: Tigecycline susceptible; TGC-NS: Tigecycline nonsusceptible; AD: Agar dilution; DD: Disk diffusion; BMD: Broth microdilution; EA: Essential agreement; CA: Categorical agreement; MI: Minor error; ME: Major error; VME: Very major error; MIC: Minimum inhibitory concentration; FDA: Food and drug administration; MHA: Mueller-Hinton Agar; MHB: Mueller Hinton broth

\section{Acknowledgements}

The authors would like to thank Dr. Kathryn Doornbos for editing the manuscript and Dr. Babak Habibi for his help with the statistical analyses. This study was supported by University of Tabriz.

\section{Authors' contributions}

SB performed the experiments, analyzed the experiment data and drafted the manuscript. $\mathrm{MH}$ is the corresponding author who designed the experiments, analyzed the experiment data and wrote the manuscript. All authors read and approved the final manuscript.

\section{Funding}

None.

\section{Availability of data and materials}

The datasets used and/or analyzed during the current study are available from the corresponding author on reasonable request.

\section{Declarations}

Ethics approval and consent to participate

The study was approved by the Research Ethics Committee of University of Tabriz. The ethics committee waived the requirement for informed consent as the bacterial isolates were obtained for research purposes from clinical samples referred to the diagnostic laboratory as part of routine care.

\section{Consent for publication}

Not applicable.

\section{Competing interests}

The authors certify that there is no conflict of interest with any financial organization regarding the materials discussed in the manuscript.

Received: 11 February 2021 Accepted: 18 June 2021

Published online: 27 July 2021

\section{References}

1. Ong CT, Babalola CP, Nightingale CH, Nicolau DP. Penetration, efflux and intracellular activity of tigecycline in human polymorphonuclear neutrophils (PMNs). J Antimicrob Chemother. 2005;56(3):498-501. https://doi.org/10.1 093/jac/dki260.

2. Zeng J, Zhang L, Gao M, Wu J, Wu H, Chen J, et al. Tigecycline treatment in an infant with extensively drug-resistant Acinetobacter baumannii bacteremia. Int J Infect Dis. 2017;61:23-6. https://doi.org/10.1016/j.ijid.2017.05.013.

3. Renteria M, Biedenbach D, Bouchillon S, Hoban D, Raghubir N, Sajben P. In vitro activity of tigecycline and comparators against carbapenem-resistant Enterobacteriaceae in Africa-Middle East countries: TEST 2007-2012. J Glob Antimicrob Resist. 2014;2(3):179-82. https://doi.org/10.1016/j.jgar.2014.03.002.

4. Sader HS, Castanheira M, Flamm RK, Mendes RE, Farrell DJ, Jones RN. Tigecycline activity tested against carbapenem-resistant Enterobacteriaceae from 18 European nations: results from the SENTRY surveillance program (2010-2013). Diagn Microbiol Infect Dis. 2015;83(2):183-6. https://doi.org/1 0.1016/j.diagmicrobio.2015.06.011

5. Beabout K, Hammerstrom TG, Perez AM, Magalhães BF, Prater AG, Clements $T P$, et al. The ribosomal S10 protein is a general target for decreased tigecycline susceptibility. Antimicrob Agents Chemother. 2015;59(9):5561-6. https://doi.org/10.1128/AAC.00547-15.

6. Sun Y, Cai Y, Liu X, Bai N, Liang B, Wang R. The emergence of clinical resistance to tigecycline. Int J Antimicrob Agents. 2013;41(2):110-6. https:// doi.org/10.1016/j.jijantimicag.2012.09.005.

7. Fluit AC, Florijn A, Verhoef J, Milatovic D. Presence of tetracycline resistance determinants and susceptibility to tigecycline and minocycline. Antimicrob 
Agents Chemother. 2005;49(4):1636-8. https://doi.org/10.1128/AAC.49.4.163 6-1638.2005.

8. Peleg AY, Adams J, Paterson DL. Tigecycline efflux as a mechanism for nonsusceptibility in Acinetobacter baumannii. Antimicrob Agents Chemother. 2007;51(6):2065-9. https://doi.org/10.1128/AAC.01198-06.

9. Hentschke M, Wolters M, Sobottka I, Rohde H, Aepfelbacher M. ramR mutations in clinical isolates of Klebsiella pneumoniae with reduced susceptibility to tigecycline. Antimicrob Agents Chemother. 2010;54(6): 2720-3. https://doi.org/10.1128/AAC.00085-10.

10. Du X, He F, Shi Q, Zhao F, Xu J, Fu Y, et al. The rapid emergence of Tigecycline resistance in blaKPC-2 harboring Klebsiella pneumoniae, as mediated in vivo by mutation in tetA during Tigecycline treatment. Front Microbiol. 2018;9:648. https://doi.org/10.3389/fmicb.2018.00648.

11. Moore IF, Hughes DW, Wright GD. Tigecycline is modified by the flavindependent monooxygenase TetX. Biochemistry. 2005;44(35):11829-35. https://doi.org/10.1021/bi0506066.

12. Hope R, Warner M, Mushtaq S, Ward M, Parsons T, Livermore D. Effect of medium type, age and aeration on the MICs of tigecycline and classical tetracyclines. J Antimicrob Chemother. 2005;56(6):1042-6. https://doi.org/1 0.1093/jac/dki386.

13. Fernández-Mazarrasa C, Mazarrasa O, Calvo J, del Arco A, Martínez-Martínez L. High concentrations of manganese in Mueller-Hinton agar increase MICs of tigecycline determined by Etest. J Clin Microbiol. 2009;47(3):827-9. https://doi.org/10.1128/JCM.02464-08.

14. Tan TY, Ng LSY, Chen DMM. Influence of different Mueller-Hinton agars and media age on Etest susceptibility testing of tigecycline. Diagn Microbiol Infect Dis. 2010;68(1):93-5. https://doi.org/10.1016/j.diagmicrobio.2010.05.001.

15. Mahon CR, Lehman DC, Manuselis G. Textbook of diagnostic microbiologye-book: Elsevier health sciences; 2018.

16. Turton JF, Woodford N, Glover J, Yarde S, Kaufmann ME, Pitt TL. Identification of Acinetobacter baumannii by detection of the blaOXA-51like carbapenemase gene intrinsic to this species. J Clin Microbiol. 2006; 44(8):2974-6. https://doi.org/10.1128/JCM.01021-06.

17. Jafari Z, Harati AA, Haeili M, Kardan-Yamchi J, Jafari S, Jabalameli F, et al. Molecular epidemiology and drug resistance pattern of carbapenemresistant Klebsiella pneumoniae isolates from Iran. Microb Drug Resist. 2019; 25(3):336-43. https://doi.org/10.1089/mdr.2017.0404.

18. Haeili M, Abdollahi A, Ahmadi A, Khoshbayan A. Molecular characterization of Tigecycline non-susceptibility among extensively drug-resistant Acinetobacter baumannii isolates of clinical origin. Chemotherapy. 2021:1-7. https://doi.org/10.1159/000515100.

19. Wiegand I, Hilpert K, Hancock RE. Agar and broth dilution methods to determine the minimal inhibitory concentration (MIC) of antimicrobial substances. Nat Protoc. 2008;3(2):163-75. https://doi.org/10.1038/nprot.2007.521.

20. FDA U. Class II Special controls guidance document: antimicrobial susceptibility test (AST) systems. US FDA: Rockville; 2009.

21. Pillar CM, Draghi DC, Dowzicky MJ, Sahm DF. In vitro activity of tigecycline against gram-positive and gram-negative pathogens as evaluated by broth microdilution and Etest. J Clin Microbiol. 2008;46(9):2862-7. https://doi.org/1 0.1128/JCM.00637-08.

22. Torrico M, González N, Giménez M, Alou L, Sevillano D, Navarro D, et al. Influence of media and testing methodology on susceptibility to tigecycline of Enterobacteriaceae with reported high tigecycline MIC. J Clin Microbiol. 2010;48(6):2243-6. https://doi.org/10.1128/JCM.00119-10.

23. Liu J-W, Ko W-C, Huang C-H, Liao C-H, Lu C-T, Chuang Y-C, et al. Agreement assessment of tigecycline susceptibilities determined by the disk diffusion and broth microdilution methods among commonly encountered resistant bacterial isolates: results from the Tigecycline in vitro surveillance in Taiwan (TIST) study, 2008 to 2010. Antimicrob Agents Chemother. 2012;56(3):14147. https://doi.org/10.1128/AAC.05879-11.

24. Jones RN, Ferraro MJ, Reller LB, Schreckenberger PC, Swenson JM, Sader HS Multicenter studies of tigecycline disk diffusion susceptibility results for Acinetobacter spp. J Clin Microbiol. 2007;45(1):227-30. https://doi.org/10.112 8/JCM.01588-06.

\section{Publisher's Note}

Springer Nature remains neutral with regard to jurisdictional claims in published maps and institutional affiliations.

Ready to submit your research? Choose BMC and benefit from:

- fast, convenient online submission

- thorough peer review by experienced researchers in your field

- rapid publication on acceptance

- support for research data, including large and complex data types

- gold Open Access which fosters wider collaboration and increased citations

- maximum visibility for your research: over $100 \mathrm{M}$ website views per year

At BMC, research is always in progress.

Learn more biomedcentral.com/submissions 